\title{
La opción por los pobres: la estética de Fidel Sepúlveda y la encíclica Laudato si
}

\section{The Option for the Poor: Aesthetics of Fidel Sepúlveda and the Encyclical Laudato si}

\author{
Felipe Espinoza Villarroel \\ Instituto de Estética, Pontificia Universidad Católica de Chile. Santiago, Chile. \\ fpespino@uc.cl
}

\section{Resumen}

La cultura tradicional fue la matriz reflexiva estética del profesor Fidel Sepúlveda, constituyéndose en modelo para una estética latinoamericana y chilena. Por otra parte, la encíclica del Papa Francisco Laudato si (2015) trascendió el ámbito estrictamente eclesial, convocando a una transversal sensibilidad con respecto al tema medioambiental y la crisis espiritual de la civilización actual. Ante esta encrucijada, Francisco recurre a la cultura tradicional como alternativa de desarrollo. Entre ambas miradas se observan coincidencias en cuanto a enfoque, temas y posibles soluciones. Este artículo plantea que ambas reflexiones se encuentran en "la opción por los pobres", fórmula acuñada por la Teología de la Liberación, asumida por Sepúlveda y Francisco desde sus coincidentes reflexiones.

Palabras clave: cultura tradicional, ecología, opción por los pobres, Teología de la Liberación.

\begin{abstract}
Traditional culture was the matrix of Fidel Sepúlveda's aesthetic reflection, matrix that constitued itself a model for a Chilean and Latin American aesthetics. On the other hand, the encyclical of Pope Francisco Laudato si (2015) became a phenomenon that transcended the strictly ecclesial world, calling for a cross-sensitivity to the environmental issue and the great spiritual crisis that crosses our civilization. Francisco resorts, among other things, to traditional culture as an alternative for future alternative development. Between both perspectives clear coincidences are observed in terms of approach, topics and possible solutions. This article purposes that both reflections meet in the formula "the option for the poor", coined by Liberation Theology, an alternative assumed by Fidel from an aesthetic reflection and by Francisco as a theological-pastoral stance.
\end{abstract}

Keywords: traditional culture, ecology, choice for the poor, Liberation Theology. 


\section{Introducción}

Fidel Sepúlveda escribió cinco autos sacramentales sobre diversos personajes -San Alberto Hurtado, Santa Teresita de Los Andes, Jesucristo- entre los que destaca San Francisco y las Creaturas (2006), con Francisco de Asís como protagonista. Además, en su obra existen indicios de una profunda relación entre su cosmovisión y la del Papa Francisco, quien, de hecho, toma su nombre del santo de Asís, figura inspiradora por su relación amorosa con la creación y las criaturas. Un ejemplo de ello es el siguiente pasaje de Voz clamante en el desierto, cuando Fidel Sepúlveda, en voz del santo de Asís, señala:

Me es fácil entenderme con la hermana agua, más fácil que con el hermano lobo, pero me es más fácil entenderme con el hermano lobo que con el hermano hombre. El hermano lobo andaba lejos de la hermana agua y no refleja ni canta, cuesta tocarlo en su entrega bondadosa, pero tocar no defrauda. Es noble y sólido y valeroso. En cuanto al hombre, que difícil es tocar algo sólido noble y valeroso. Cuanto más alto y poderoso y más sólido y noble y valeroso parece, más intocable e inasible es, cada hombre no es uno sino muchos por entre los cuales se transita sin toparse con ninguno. Desierto está el mundo (94-5).

El último verso del fragmento destaca por su carácter profético y decidor, constituyendo una suerte de diagnóstico, que resuena desoladoramente actual a la luz de lo que plantea Francisco en Laudato si (2015). Al inicio de dicha encíclica, explicita el origen del título de este texto, Alabado sea, tomado de una fórmula franciscana. Por tanto, a lo largo de este artículo se planteará, en primer lugar, un paralelo entre los principales planteamientos de Fidel Sepúlveda en torno al tema ecológico desde una perspectiva estética, y algunas de las afirmaciones más relevantes que en esta línea el Papa Francisco expresa en Laudato si con el fin de evidenciar la coincidencia de ambas visiones. Posteriormente, se indaga en las razones posibles de esta coincidencia, a partir de la referencia que aparece en ambos de la expresión acuñada por la Teología de la Liberación y la Iglesia latinoamericana de los años sesenta: "la opción preferencial por los pobres".

\section{La estética de Fidel Sepúlveda}

Las reflexiones del profesor Sepúlveda podemos concebirlas, a grandes rasgos, como parte de una lectura estética de la cultura popular chilena y latinoamericana. Cuando Sepúlveda habla de estética la concibe enraizada en nuestra realidad cultural popular, alejada de un desarrollo meramente conceptual y encarnada en un ethos particular. En otras palabras, su obra es el esfuerzo por posicionar al ámbito de lo latinoamericano y de lo chileno como un lugar privilegiado para hablar y desarrollar una estética 
particular. Él mismo se referirá a ella como "una estética del otro mundo y del otro modo" (Patrimonio 107): el mismo "mundo al revés" que nuestros poetas populares plantean como uno de sus principales temas o fundaos, donde se cambian los órdenes establecidos y se invierten las jerarquías, al modo del carnaval y la fiesta popular. Puntualmente, el canto a lo poeta fue para Fidel Sepúlveda uno de sus principales desarrollos teóricos: veía allí la posibilidad de un lugar para desarrollar una estética no tributaria de modelos foráneos en su integridad.

Más allá de estas referencias culturales, la estética de Sepúlveda puede leerse también como una crítica radical a algunas nociones del arte occidental que se dan por sabidas y constituidas de suyo (167). Afirmaciones como "la historia del arte y de la estética está por hacerse y debiera comenzar por situar al folklore como arte modélico" (167) son un ejemplo de esto, reafirmado en cómo este arte modélico, la cultura tradicional, genuino "arte-vida", constituye una posibilidad de lugar para pensar el arte, las obras, la estética y, en general, la cultura desde Latinoamérica. Como latinoamericanos debemos evitar transferir directamente ciertas nociones de la cosmovisión ilustrada europea hacia nuestra realidad y, antes bien, adoptar y adaptar tales conceptos, encarnándolos a partir de un arraigo genuino y original.

Para Sepúlveda se puede hablar de una identidad, y la estética, justamente, es un camino para ello (28). Lo identitario fue, tal vez, el gran tema de su pensamiento, tema que investigó y pensó hasta sus últimos días de vida. Precisamente en la línea de pensarnos como chilenos y latinoamericanos la estética se constituye como un camino posible para sondear una identidad que a ratos es difusa, esquiva y difuminada. Sumado a esto, aparecen la creación de obras y estilos de vida que, para Sepúlveda, serán el arte por excelencia, el arte de vivir, vinculado con la idea de una cultura tradicional en tanto camino hacia un desarrollo alternativo.

\section{La novedad de Laudato si}

Al comienzo de la encíclica el Papa Francisco aclara que, desde Juan xxIII en adelante, el tema medioambiental ha sido tratado por otros pontífices en diversos documentos pastorales. Sin embargo, y he allí su mayor novedad, nunca había sido abordado con la profundidad y el enfoque que él le da. No es casual que Laudato si fuese por semanas uno de los libros más vendidos, fenómeno completamente inédito para un documento oficial del Vaticano. El texto fue leído y acogido por ateos, cristianos y agnósticos convirtiéndose en un mensaje ecuménico-transversal. Dicha recepción es señal de que el texto toca problemáticas que van más allá de los credos e iglesias, es decir, aborda temas que atañen a la civilización completa.

En Laudato si, el Papa Francisco sostiene que resultan inseparables la preocupación por la naturaleza, la justicia con los pobres, el compromiso social y la paz interior (10). Tal enfoque multisistémico de esta encíclica coincide en varios aspectos con el 
pensamiento de Fidel Sepúlveda. No se trata solamente del tema medioambiental porque, si hablamos de medioambiente, necesariamente aparecerán otros tópicos relacionados. Dicha perspectiva amplía la noción de ecología hacia una "ecología integrada", perspectiva que incorpora dimensiones humanas y sociales. Es justamente allí donde se abre la posibilidad de un diálogo entre este texto, Fidel Sepúlveda y la "mirada hacia los no mirados", es decir, desde la íntima relación entre la fragilidad del planeta y los pobres o, en palabras de Francisco, los “descartables". Además, el principio del bien común se convierte en un llamado a la solidaridad y en una opción preferencial por los más desamparados: allí se encuentran la reflexión de Fidel Sepúlveda con Laudato si.

\section{La estética de Fidel Sepúlveda y Laudato si: elementos para un encuentro}

En lo que sigue se ensayará establecer un diálogo entre ambos planteamientos guiado desde ciertas preguntas. La primera podría ser en torno a cómo vive el hombre contemporáneo. Entre sus múltiples escritos, Sepúlveda plantea: "El mundo actual tiene oído, pero no escucha, tiene ojos y no ve, lo rodea la ilusión, la corrosión, la destrucción y el desmantelamiento del mundo en que habita y hace oídos sordos se echa tierra y pseudo razones a los ojos" (“Ética, estética, ecología” 86). En tanto el Papa en Laudato si, sostiene: "Hay un modo de entender la vida y la acción humana que se ha desviado, y que contradice la realidad hasta dañarla” (79). El diagnóstico realizado, uno desde la academia, otro desde el pontificado y la experiencia pastoral, empieza a encontrarse en una aproximación común respecto de la vida actual, incluso aunque ambos escritos se distancien por veinticinco años.

Otro tema en común es el de la crisis de la modernidad, abordado profusamente y desde distintas veredas disciplinarias. Al respecto, plantea Sepúlveda: "La modernidad ha concentrado buena parte de su energía en correr las marcas de la frontera; esto ha llevado a privilegiar la originalidad entendida como novedad, y la novedad como la capacidad de hacer noticia. Se es cuanto más distante y opuestos se es al otro" (Patrimonio 57). Es el paradigma de la competencia y la oposición donde no se afirma la identidad por pertenencia, sino debido a que surge por oposición: soy más cuanto más me separo y más distante me hago del otro. A esto se agrega el concepto de originalidad como hacer noticia y no ir al origen (57).

Por su parte, el Papa señala: "El inmenso crecimiento tecnológico no estuvo acompañado de un desarrollo del ser humano en responsabilidad, en valores, en conciencia. Cada época tiende a desarrollar una escasa autoconciencia de sus propios límites" (82). Con ello aparece otra idea ampliamente analizada por la filosofía contemporánea y las ciencias sociales desarrolladas a lo largo de la modernidad: creamos y poseemos múltiples medios, pero ¿para qué fines? Al respecto, señala Fidel: "La 
hipertrofia del tener en la sociedad contemporánea conduce a una sed y a un hambre que no pueden ser satisfechos con lo que da el tener. Así, el hombre de la era actual no disfruta de paz, de armonía, de felicidad. Sumido en la oferta de bienes materiales como único horizonte se desespera por no tener todo lo que desea" ("Ética, estética" 91). En tanto, Francisco planteará: "Tal paradigma -el técnico económico- hace creer a todos que son libres mientras tengan una supuesta libertad para consumir. La humanidad postmoderna no encontró una nueva comprensión de sí misma que pueda orientarla y esta falta de identidad se vive con angustia. Tenemos demasiados medios para unos escasos y raquíticos fines" (155). Aquí reaparece el tema identitario. De algún modo, los múltiples y profusos medios para unos escasos fines es un punto clave en la reflexión que atraviesa ambos pensamientos, ya que se dirigen en el mismo sentido crítico.

Desembocamos así en una "humanidad adelgazada": "el hombre de la era posindustrial ha recortado su mundo por lo alto, por lo ancho y por lo hondo, se ha unidimensionado, sus necesidades fundamentales no desaparecen, están ahí postergadas y cuanto mayor es esta postergación, más lesión, más deterioro se produce al nivel de la economía esencial del hombre" (Sepúlveda, "Ética, estética" 92). En esa misma línea sostendrá Francisco: "El modo como la humanidad de hecho ha asumido la tecnología y su desarrollo junto con un paradigma homogéneo y unidimensional, lo que interesa es extraer todo lo posible de las cosas con la imposición de la mano humana, el ser humano y las cosas han dejado de tenderse amigablemente la mano para pasar a estar enfrentados" (83).

Otra reflexión relevante de Sepúlveda estriba en cómo reúne el sentido con el concepto -muy en boga- de calidad de vida: "El sentido de la existencia debe considerar la calidad de vida, pero la calidad de vida no tiene su fundamento primero y último en el ingreso per cápita, se afinca en el ingreso a mi ser de la irradiación de vida que emite lo humano, lo cósmico, lo divino que puebla todo el universo" (92). En tanto, Francisco señalará: "Se trata de redefinir el progreso. Un desarrollo tecnológico y económico que no deja un mundo mejor y una calidad de vida integralmente superior no puede considerarse progreso" (148). Esto último aparece como una crítica directa a la modernidad, que plantea el progreso como principal consigna programática. Sin embargo, y observando nuestra situación actual, vemos que la idea de progreso habría que redefinirla pues si se mantiene en los términos actuales el panorama no resulta en absoluto auspicioso, menos cuando el planeta se encuentra en la crítica situación actual, precisamente, por la misma idea de progreso.

Uno de los puntos más acuciantes de la confluencia entre estas dos miradas es el paralelo entre la "cultura de supermercado" que plantea Fidel y lo que dice el Papa respecto de los "descartables": "Diera la impresión de que el ideal del hombre contemporáneo fuera erradicar de la sociedad la búsqueda de otros bienes que no sean los materiales, la imagen de su ideal sería instalar su morada en el centro del supermercado de modo de tener al alcance de la mano todos los productos, es el mundo convertido 
en supermercado, de productos terminados" (92). En tanto, Francisco sostiene: "Es la misma lógica del usa y tira que genera tantos residuos, solo por el deseo desordenado de consumir más de lo que realmente se necesita" (96).

En una encíclica anterior, Evangelii gaudium (2013), ya había hablado de esta "cultura del descarte", en clara sintonía con la cultura del supermercado que enunciaba Fidel varios años atrás. Dicha cultura se encuentra en total desarmonía con la cosmovisión de la cultura tradicional donde los productos no están terminados y lo desechable no resulta concebible.

Ante tal diagnóstico de la cultura del supermercado, la abundancia de medios y la escasez de fines, se plantea como alternativa la experiencia comunitaria del encuentro. En el encuentro, señala Sepúlveda, se revela la riqueza y sentido vital de lo existente humano y mundano. Tal sentido profundo de la ecología aparecerá de manera incipiente en "Materiales para una estética", texto del año 1982, y de modo más decidido, a principios de los noventa en el ya citado "Ética, Estética y Ecología".

En Laudato si, Francisco sostiene: "Muchas personas en estas condiciones - referido al hacinamiento, al anonimato, al desarraigo a la violencia- son capaces de tejer lazos de pertenencia y de convivencia que convierten el hacinamiento en una experiencia comunitaria donde se rompen las paredes del yo y se superan las barreras del egoísmo" (116). La experiencia comunitaria del encuentro le otorga riqueza y sentido vital a la vida humana. Se trata entonces de abrirnos al otro para aspirar a un desarrollo otro: "Hay una vía por la cual avanzar en la experiencia del ser humano es la experiencia de hacer con el otro, ser con el otro que hay en mí, que es el otro que yo soy. Yo soy en la medida que los otros son, yo soy los otros, con los otros; los otros no son sin mí. Algo de los otros no es cuando yo no soy" (Sepúlveda, Patrimonio 57). En términos poético-lúdicos, Sepúlveda plantea la radicalidad de la otredad. Paralelamente, señalará Francisco: "La capacidad de salir de sí hacia el otro, sin ellas no se reconoce a las demás criaturas en su propio valor, no interesa cuidar algo para los demás, no hay capacidad de ponerse límites. Cuando somos capaces de superar el individualismo realmente se puede desarrollar un estilo de vida alternativo y se vuelve posible un cambio importante" (158).

Además, el Papa Francisco ve la posibilidad de un cambio y un desarrollo alternativo en el encuentro con el otro, encuentro al que referirá Sepúlveda en tanto una "filosofía de la vinculación": la relación del yo con los otros, el mundo y lo Otro -con mayúscula-, constituyendo otro de los puntos de encuentro más estimulantes entre ambos planteamientos.

Con respecto al tema del arte-vida, Sepúlveda señala: "El arte de vivir de la cultura popular chilena toma cuerpo y espíritu con el valor de la solidaridad, en esta se hace presente una relación de parentalidad entre el hombre y los otros hombres, entre el hombre y el mundo, entre esto y lo otro, natural y sobrenatural" (Patrimonio 59). En Laudato si, Francisco señalará: “El análisis de los problemas ambientales es inseparable del análisis de los contextos y de la relación de cada persona consigo 
misma que genera un determinado modo de relacionarse con los demás y con el ambiente" (110).

De este modo, la filosofía de la vinculación se encuentra en la base de ambos pensamientos: la relación entre el yo y el otro, el entorno y lo Otro, lo trascendente. Es una visión sistémica que a través de una mirada integral coinciden en diversos puntos ambos planteamientos. Sin embargo, no es esta una relación lineal, más bien exhibe las relaciones implícitas e inadvertidas entre ambas reflexiones, las cuales aluden a una estética común.

Al respecto, Sepúlveda aporta una imagen sugerente: "Para el sujeto estético una gota de agua y un grano de arena, un estambre y una espora convocan el afán de ser del universo" ("Ética, estética" 87). En paralelo, se afirma en Laudato si: "Así como los distintos componentes del planeta están relacionados entre sí, también las especies vivas conforman una red que nunca terminamos de conocer y comprender" (107). Lo anterior se relaciona no solo con la mencionada mirada sistémica, sino que también con una relación humilde respecto de la creación, es decir, una que concibe que siempre existirá algo que se escape de nuestro rango de comprensión, pues el sistema resulta siempre más amplio y complejo que el limitado entendimiento humano.

En este punto resulta pertinente reparar en la idea de Sepúlveda referente a la cultura en tanto "cultivo y crianza": "La cultura aquí la entendemos como cultivo y crianza del hombre y su circunstancia” (Patrimonio 56). La mirada estética de Sepúlveda es también un esfuerzo por redefinir algunos de los conceptos más usados en el ámbito de la antropología y la filosofía de la cultura. Lo cultural se manifiesta a través de formas comunitarias de expresión, pensamiento y acción, reveladas a través de dichas formas. Señala Francisco: "Un modo de vida que durante mucho tiempo ha otorgado identidad cultural y un sentido de la existencia y de la convivencia. La desaparición de una cultura puede ser tanto o más grave que la desaparición de una especie animal o vegetal" (113-4). Tal reflexión resulta innovadora y rupturista, pues instala en un mismo nivel a lo natural con lo cultural: ello convierte a la encíclica en un texto - si cabe la expresión- "medioambiental-cultural". Al colocar lo cultural a la par con lo medioambiental reaparece la idea de cultura en tanto cultivo. De ese modo, la metáfora terrestre sintoniza con la idea de que cuando una cultura desaparece es también una especie la que se extingue, es decir, una realidad cultivada por años, fraguada con el paso del tiempo.

Así, la cultura de las raíces nos conduce al tema identitario, pues cuando se la concibe desvinculada de ellas y de las identidades locales lo que se ve más afectado es la calidad de vida (Sepúlveda, Patrimonio 105). El concepto de arraigo, tan presente en la estética de Sepúlveda y vinculado a lo cultural-identitario, también se encuentra en Francisco: "La instancia local puede ser una diferencia, pues allí se puede generar una mayor responsabilidad, un fuerte sentido comunitario, una especial capacidad de cuidado y una creatividad más generosa, un entrañable amor a la propia tierra. Así como se piensa en lo que se deja a los hijos y a los nietos" (137). 
El concepto de arraigo y de atender a las raíces va unido a la idea de cuidar aquello que se hereda para las futuras generaciones. En esta línea, se considera a la acción comunitaria como aquellos tejidos sociales locales que van haciendo y sostienen finalmente a una comunidad. Lo sostendrá Francisco a lo largo de todo el texto: lo local puede hacer la diferencia y a partir de esto es que la cultura tradicional puede concebirse como alternativa de desarrollo.

En sintonía, plantea Sepúlveda que en la acción comunitaria: "Hay épocas y escuelas que con frecuencia operan por oposición a lo que ha sido la época inmediatamente anterior. Al lado de estos aportes, invisibles -eso es importante- muchas veces opera otra fuerza decisiva: la acción comunitaria de creación y crítica" (Patrimonio 57). En tanto, Francisco señala: "La conversión ecológica que se requiere para crear un cambio duradero es también una conversión comunitaria" (166).

De esta forma, convergen la conversión ecológica con la comunitaria, pues son ámbitos intrínsecamente relacionados, los cuales se distorsionan al concebirse separadamente. Esto último pues aquellas "Asociaciones que intervienen a favor del bien común desarrollan o recuperan vínculos y surgen nuevo tejido social local, esto incluye el cultivo de una identidad común, de una historia que se conserva, de una historia que se trasmite" (174). Dicha idea de las comunidades como "tejido" resulta sugerente: es una invitación a concebir la cultura como una trama a leer, un texto, que retoma la etimología de esta palabra vinculada, precisamente, a lo textil. Las comunidades resultan así macrotejidos a ser leídas, sostenidas por los vínculos locales y populares.

Sepúlveda comprende a la cultura popular en tanto "acontecer de la tradición" (Patrimonio 56). El concepto de lo popular resulta a menudo equívoco y engorroso, difícil de conceptualizar; sin embargo, sale al paso de esta problemática al vincular lo popular con la tradición, entendiendo así cultura tradicional y popular como equivalentes. La tradición no es una visión anquilosada de la realidad, sumergida en la nostalgia de un pasado que no volverá, más bien constituye un metabolismo ágil que selecciona día a día lo vital para el presente (56). Allí concurre la comunidad lúcida que va discerniendo sus propios materiales, donde lo viejo y lo nuevo crecen juntos hacia una validez llena de vida (56). De este modo, resulta interesante que Fidel considere a la tradición filosófica europea -en este caso la hermenéutica fenomenológica del siglo $\mathrm{xx}$ - en tanto respaldo teórico plantear la posibilidad de una estética popular surgida desde la cultura chilena. ${ }^{1}$

Con respecto a una ecología que no solo se reduce a la temática ambiental, el Papa Francisco incorpora la noción de patrimonio: "La cultura no solo en el sentido

1 La presencia de la hermenéutica fenomenológica del siglo xx puede advertirse en varios lugares de la obra de Sepúlveda. Es más, utiliza conceptos de autores como Ricoeur y Heidegger para conceptualizar la cultura tradicional, otorgándole de ese modo un estatus epistemológico similar al de la denominada alta cultura. Al respecto, ver "Reflexión sobre la cultura chilena" y "Estética del entorno del ser chileno" en Patrimonio, identidad y creatividad; El canto a lo poeta a lo divino y a lo humano: análisis estético antropológico y antología fundamental y El cuento tradicional chileno: estudio estético y antropológico y antología esencial. 
de los monumentos del pasado, sino especialmente en su sentido vivo, dinámico y participativo que no puede excluirse a la hora de repensar la relación del ser humano con el ambiente" (112). De esta manera, bajo tal concepto de ecología no podemos marginar a la cultura tradicional, entendida como instancia viva, dinámica y participativa.

Sepúlveda también realiza una reflexión interesante con respecto a la noción de originalidad al vincularla al "origen" antes que a la rareza o la novedad. La originalidad de los relatos del acervo popular, por ejemplo, consistiría en un escuchar y aprender de allí el origen, raíz y semilla de lo humano. Tal reflexión no dista de lo que propone el Papa Francisco: "hemos sido infieles al tesoro de sabiduría que debíamos custodiar. Es precisamente el regreso a sus fuentes lo que permite responder mejor a las necesidades actuales" (153). Es la vuelta hacia lo tradicional, no como retorno nostálgico a un pasado anhelado, más bien en tanto lo tradicional posee las claves para leer el presente y el futuro. Es "el viaje a la semilla" dirigido a replantear el desarrollo actual y el futuro.

El concepto "arte-vida" de Sepúlveda se vincula a lo anterior como un saber leer, entre otras cosas, la naturaleza: "La vida no es viable sino como arte de vivir... la sabiduría para leer al interior de la naturaleza humana aquello que en su esencia la atiende y satisface” (Patrimonio 59). En sintonía con ello, dirá el Papa: "A la continua aceleración de los cambios de la humanidad y del planeta se une hoy la intensificación de ritmos de vida y de trabajo, en eso que algunos llaman 'rapidación'. Si bien el cambio es parte de la dinámica, la velocidad que las acciones humanas le imponen hoy contrasta con la natural lentitud de la evolución biológica” (17).

Se alude así al evidente contraste con lo que ocurre en las grandes urbes entre los ritmos naturales y los tiempos impuestos para la cotidianeidad. Esto se relaciona con la sabiduría del arte de vivir a la que alude Sepúlveda: la lucidez y sapiencia para leer en la naturaleza humana sus ritmos y ciclos genuinos. Es decir, no imponer un modo de relación con la naturaleza, tal como ha ocurrido hasta la actualidad, imposición que es causa del actual desastre que se vive como civilización.

Las nociones de cambio y tiempo subyacen en la cultura tradicional. El cambio no es negado, por el contrario, la acción de la comunidad resulta permanente y operante, incluso constituye una zona compleja que requiere de lenta gestación, de un ritmo que lleva inscrito en su propia dinámica. Sin embargo, dirá el Papa Francisco, dentro del esquema del rédito, no hay lugar para los ritmos de la naturaleza, sus tiempos de degradación y regeneración, tampoco para la complejidad de los ecosistemas, que pueden ser gravemente alterados por la intervención humana (146).

$\mathrm{El}$ asombro y el misterio también son temas que vinculan ambas reflexiones. Al respecto, Sepúlveda señala que debemos contar con el misterio (Patrimonio 26) y el Papa planteará que una reflexión ecológica actual debiese ver el mundo no como un problema a resolver, sino en tanto misterio gozoso que contemplamos con jubilosa alabanza (12). 
La estrecha vinculación entre ética, estética y ecología integra la propuesta de ambos pensamientos. Francisco señala: "Si se reflexiona con ese marco cerrado, con el marco solamente científico desaparece la sensibilidad estética, la poesía, aun la capacidad de la razón para percibir el sentido y la finalidad de las cosas" (152). En tanto, para Sepúlveda, la estética constituye la fuente primordial de toda ecología, una que no puede considerarse desvinculada del desarrollo ético, entendido como la manera en que nos relacionamos con nosotros mismos, los otros, el entorno y lo trascendente.

\section{La opción por los pobres: lugar para el encuentro de dos miradas}

La pregunta es, entonces, más allá de las sintonías y coincidencias entre ambos planteamientos, cuál sería el lugar donde se encuentran estos dos planteamientos. El pensamiento de Sepúlveda y el de Francisco convergen en la fórmula que acuñó la Teología de la Liberación en los años sesenta, a la que Francisco vuelve en Laudato $s i$, esta es, "la opción por lo pobres".

Tanto Fidel Sepúlveda -desde la teoría estética y su reflexión identitaria-como Francisco -en su encíclica y otros textos- realizan una opción por los pobres, por la cultura de la marginalidad, es decir, por los excluidos y no mirados por la sociedad. Dicha opción compartida hace que estos pensamientos coincidan y converjan en temas medulares y fundamentales; en otras palabras, es desde y a partir de la opción por los pobres que tal coincidencia de miradas resulta posible.

Latinoamérica, dice Sepúlveda, es "cultura de la precariedad": de allí que no tenga lugar "la opción del supermercado", pues las necesidades son infinitas y los medios escasos: se tiene que hacer "todo" y "de todo" (Patrimonio 58). En tanto, la reflexión de Francisco sostiene que un planteo ecológico que no considera a los pobres ni muestra un compromiso con la sociedad y una espiritualidad, no es "verdadera" ecología (10). El clamor de la tierra es el clamor de los pobres, los mismos que vieron Fidel y Francisco desde sus distintas perspectivas.

Al inicio de su pontificado el Papa Francisco recalcó la importancia de una "iglesia en salida, una iglesia pobre y para los pobres", con lo cual causó un gran impacto a nivel eclesial y social. A esto se une la idea de detener el paso y dejar de lado la ansiedad -dejarse guiar por los ritmos naturales- para escuchar y renunciar a las urgencias personales y acompañar al que quedó al margen, propuesta alegorizada en la parábola del buen samaritano (Lucas 10, 25-37).

2 Al respecto ver Julio Lois, Teología de la liberación: la opción por los pobres; Segundo Galilea et al. Enrique Alvear y la opción por los pobres: reflexiones y testimonios y Leonardo Boff et al. Sobre la opción por los pobres. 
Sepúlveda en tanto hace hincapié en lo que denomina "la riqueza de la pobreza", es decir, la pobreza es "pobre", entre comillas, porque tal carestía es muy rica y tiene un enorme potencial para nutrir a los no marginados o privilegiados. Creatividad y solidaridad operan aquí de manera conjunta, y destacan la gran veta creativa de la cultura tradicional: medios escasos y necesidades infinitas son los materiales para la vida y la subsistencia: con ellos es posible también realizar los denominados milagros.

La vida, entonces, es concebida como arte de vivir. La creatividad, por su parte, nos habla de un arte-vida que comprende la existencia en tanto obra de arte por realizar. Tal proceder es comprobable con las distintas expresiones de las culturas tradicionales, conformadas por excluidos que habitan las denominadas poblaciones periféricas. El teólogo argentino Juan Carlos Scannone sostiene esa idea: "La sabiduría de los pobres en sus relaciones solidarias en la belleza de sus fiestas y sus símbolos, muchas veces la creatividad comunitaria muestra una gratuidad irreductible a la racionalidad" (226). Fidel Sepúlveda insistió en la gratuidad que caracteriza a la cultura tradicional: la idea de una solidaridad creativa se concretiza en profusas expresiones de las distintas comunidades -como la minga o las ollas comunes-donde prima la experiencia del compartir y acompañar al otro.

Ronaldo Muñoz, destacado teólogo chileno de la Teología de la Liberación, plantea que el verdadero milagro ocurre en el operar de la cultura tradicional, idea similar en la que reparan Sepúlveda y el Papa Francisco:

El asombro radical que nos produce ese verdadero milagro de la supervivencia humana y la solidaridad de la gente. Uno se pregunta cómo puede la gente seguir viviendo, cómo pueden seguir haciendo bromas, riendo y cantando. Cómo la gente es capaz de compartir lo que no tiene, cómo son capaces de cantar y bailar, de reflexionar juntos, de crear. Es un verdadero milagro (43).

De esta forma, algunas de las reflexiones de la Teología de la Liberación se entroncan con el arte-vida que plantea Sepúlveda. Jorge Costadoat, teólogo jesuita dedicado a reflexionar sobre la pertinencia y actualidad de una teología liberadora, repara en el rol que en ello posee la creatividad de los pobres:

Para esta teología los pobres no solo han de ser objeto de caridad y de justicia, ellos deben ser considerado sujetos que inventan un mundo nuevo, con escasos materiales, pero con la comprensión vital de un evangelio que ha sido anunciado a ellos antes que a nadie. El mayor aporte de la Teología de la Liberación, y de aquí su futuro, estriba en creer en la creatividad de los pobres ("Actualidad y Futuro").

La actualidad y pertinencia que pueda tener la Teología de la Liberación el día de hoy pasa por la creatividad de los pobres, que podríamos denominar como "ecología humana de la pobreza": "Es admirable la creatividad, la generosidad de personas y grupos que son capaces de revertir los límites del ambiente modificando los efectos adversos, los condicionamientos, cualquier lugar deja de ser un infierno y se 
convierte en el contexto de una vida digna" (Francisco, 115-6). En tanto, Ronaldo Muñoz repara en: "Cómo son capaces los cesantes y demás vecinos de esa población de inventar cosas increíbles; allí se juntan, cuentan chistes, cantan, comentan la situación, improvisan poesías, pequeñas representaciones, abren juntos un espacio de cuatro horas de vida humana" (44). También reflexionando en torno a la pertinencia y posible rol de la Teología de la Liberación en la actualidad, señala Costadoat: "En 40 años de Teología de la Liberación, ante el despliegue arrollador del neoliberalismo y tras el fracaso de los modelos socialistas estatistas, revalora el desarrollo como modelo de comprensión y de modificación de la realidad social. No ya en los términos de los años 50, pero sí en la perspectiva del PNUD, un desarrollo integral" ("La Teología de la Liberación"). El mismo concepto de desarrollo lo va a considerar Sepúlveda cuando plantea: "La noción de desarrollo ha adquirido su pleno sentido al afirmar la necesidad de tener en cuenta no solo la fuerza del trabajo de los hombres, sino también su identidad cultural, en la que se funda su visión del mundo" (Patrimonio 18).

Entonces, ¿quiénes son, hoy por hoy, los pobres? Precisamente aquellos que los procesos de globalización marginan y desintegran. Por tanto, mientras la exclusión no se acabe, la Teología de la Liberación continuará teniendo plena vigencia. Leonardo Boff, teólogo brasileño de esta corriente, señala en plena sintonía con lo planteado por el Papa Francisco: "Hoy gritan las aguas, los bosques, los animales, es toda la tierra la que grita. Dentro de la opción por los pobres y contra la pobreza debe ser incluida la tierra y todos los ecosistemas. La tierra es el gran pobre que debe ser liberado". La tierra es, entonces, el pobre por el que tendríamos que optar y, más allá si el Papa es propiamente un teólogo de la liberación, el epicentro de su pensamiento es la opción por los pobres, los marginados: sean estos niños, ancianos, mujeres, indígenas, discapacitados, jóvenes, inmigrantes.

Es por esa vigencia que Costadoat señala que el libro de Gustavo Gutiérrez La Teología de la Liberación de 1972 ya no sirve tal como se escribió, pero resulta indispensable escribirlo de nuevo ("La Teología"). Pensamientos como el de Fidel Sepúlveda y lo planteado por el Papa Francisco en Laudato si se inscriben en esa línea de reescribir una teología que libere a la humanidad no de las ataduras de los años sesenta, sino de las que hoy en día impiden un desarrollo de ciudadanos reflexivos, conscientes e integrales.

\section{Referencias}

Boff, Leonardo. "Si no tenemos utopías, nos empantanamos en los intereses individuales". Clarín 24 de agosto 2008. Impreso.

---. et al. Sobre la opción por los pobres. Santiago: Rehue, 1992. Impreso. 
Costadoat, Jorge. "Actualidad y Futuro de la Teología de la Liberación”. <http://jorgecostadoat.cl/wp/actualidad-y-futuro-de-la-teologia-de-la-liberacion-2/>. Fecha de ingreso: 8 de noviembre de 2017. Web.

---. "La Teología de la Liberación 40 años después" El Mostrador 29 de noviembre de 2012. <http://www.elmostrador.cl/noticias/opinion/2012/11/29/la-teologia-dela-liberacion-40-anos-despues/>. Fecha de ingreso: 19 de enero de 2017. Web.

Francisco. Laudato Si. Carta encíclica del santo padre Francisco sobre el cuidado de la casa común. Madrid: Romana, 2015. Impreso.

Galilea, Segundo et al. Enrique Alvear y la opción por los pobres: reflexiones y testimonios. Santiago: Ediciones Paulinas, 1985. Impreso.

Lois, Julio. Teología de la liberación: la opción por los pobres. Madrid: IEPALA, 1988. Impreso.

Muñoz, Ronaldo. Dios de los cristianos. Madrid: Ediciones Paulinas, 1987. Impreso.

Scannone, Juan Carlos. Irrupción del pobre y quehacer filosófico: hacia una nueva racionalidad. Comp. Juan C. Scannone y Marcelo Perine. Buenos Aires: Borum, 1993. Impreso.

Sepúlveda, Fidel. Patrimonio, identidad, tradición y creatividad. Santiago: Centro de Investigaciones Diego Barros Arana, 2010. Impreso.

---. "Materiales para una estética del entorno" Aisthesis 14 (1982). 11-23. Impreso.

---. "Ética, estética, ecología”. Aisthesis 25-26 (1992-93). 85-95. Impreso.

---. Voz clamante en el desierto: cinco autos sacramentales. Santiago: Ediciones Universidad Católica de Chile, 2006. Impreso.

Recibido: 27 enero 2017 Aceptado: 30 septiembre 2017 